\title{
Theoretical and Experimental Study of Harmonically Modelocked Fiber Lasers for Optical Communication Systems
}

\author{
Moshe Horowitz, Curtis R. Menyuk, Thomas F. Carruthers, and Irl N. Duling, III
}

Invited Paper

\begin{abstract}
We study theoretically and experimentally actively modelocked fiber lasers that are used in high repetition rate optical communication systems. Using an innovative numerical technique and a reduced model, we have found that the laser can operate in four different operating regimes when the laser intensity was changed; three of the regimes were experimentally observed in a laser with a sigma configuration. An excellent quantitative agreement between the theoretical and the experimental results was obtained. The use of dispersion management in the sigma laser was found to significantly improve the laser performance.
\end{abstract}

Index Terms-Modelocked lasers, nonlinear optics, optical fiber lasers, pulsed lasers, solitons.

\section{INTRODUCTION}

$\mathbf{M}$ ODELOCKED erbium-doped fiber lasers are compact and environmentally stable and can generate dropout-free ultrashort pulses with a very high repetition rate, a large extinction ratio, and a very low phase noise. These features make them particularly well suited for studying the transmission of return to zero (RZ) pulses, e.g., solitons, at data rates where are no other short pulse sources that are as clean available. Additionally, those sources have application in optical high-data-rate analog to digital conversion. An actively modelocked laser with a sigma configuration has been demonstrated to generate a stable train of 1-ps pulses with a repetition rate of $10 \mathrm{GHz}$ and a pulse dropout ratio of less than $10^{-14}$ [1]. This laser has been used in repeaterless

Manuscript received February 17, 2000; revised August 10, 2000. This work was supported by DOE, NSF, the Division for Research Funds of the Israel Ministry of Science, and by the Office of the Chief of Naval Research.

M. Horowitz is with the Department of Electrical Engineering, Technion-Israel Institute of Technology, Haifa, 32000 Israel (e-mail: eermoshe@ee.technion.ac.il), and is also with the Department of Computer Science and Electrical Engineering, University of Maryland Baltimore County, Baltimore, MD 21250 USA.

C. R. Menyuk is with Department of Computer Science and Electrical Engineering, University of Maryland Baltimore County, Baltimore, MD 21250 , USA, and is also with the Laboratory for Telecommunication Sciences, Adelphi, MD 20783-1197 USA

T. F. Carruthers is with the Optical Sciences Division, Naval Research Laboratory, Washington, DC 20375-5338 USA.

I. N. Dulling was with the Optical Sciences Division, Naval Research Laboratory, Washington, DC 20375-5338 USA. He is now with Optinel Systems, Columbia, MD 2104 USA.

Publisher Item Identifier S 0733-8724(00)09820-0. transmission of 10-Gb/s data over $296 \mathrm{~km} \mathrm{[2]} \mathrm{and} 4 \times 10$ $\mathrm{Gb} / \mathrm{s}$ wavelength-division multiplexed data over $235 \mathrm{~km} \mathrm{[3].} \mathrm{A}$ similar fiber laser has been recently used for transmitting 20 $\mathrm{Gb} / \mathrm{s}$ of data to more than $20000 \mathrm{~km}$ [4]. The sigma laser has also exhibited an extremely low measured timing jitter of less than $10 \mathrm{fs}$ over a $100 \mathrm{~Hz}-1 \mathrm{MHz}$ frequency interval [5].

Due to the stringent requirements imposed upon lasers that are used in optical communication systems, it is important to develop theoretical models that enable the user to accurately analyze laser performance. Such models will give researchers the means to better understand the physics of the laser and to control and optimize its performance. Prior work for studying actively modelocked fiber lasers or storage rings [6], [7] has been largely based on solving the Ginzburg-Landau equation, modified to include amplifier filtering and active modelocking (This equation is also referred to as the master equation of modelocking). This work, which is based on earlier pioneering work by Haus [8] and by others [9], includes a number of simplifying assumptions. The most important assumption in the reduced model is that 1) every pulse in the laser cavity is the same. This assumption, which is certainly false in general, makes it impossible to study important dynamic effects in harmonically modelocked fiber lasers and to find the precise limits on the stable operating regime; however, it is a reasonable assumption when studying an already-established stable pulse train. Other assumptions are: 2) the pulse change is small at any fixed point in the laser from one roundtrip to the next; 3 ) the bandwidth of the pulse is small compared to the bandwidth of the gain medium and/or the optical filtering; and 4) the time duration of the pulse is much shorter than the period of the modelocking. Additionally, to use soliton perturbation theory, one must assume that 5) the pulse shape remains nearly hyperbolic-secant during its roundtrip through the laser. These assumptions enable one to obtain analytical results and to find out explicitly the dependence of the generated pulses on the cavity parameters. In order to obtain a good quantitative agreement between the theory and experiments, however, one should use a more comprehensive approach that models all the major physical phenomena that affect the laser behavior.

One of the important effects that cannot be accurately modeled using a single pulse analysis is pulse dropout. Since fiber lasers for optical communication systems operate at high repetition rates, they are harmonically modelocked so that 
many pulses simultaneously propagate inside the cavity. A major problem with this mode of operation is pulse dropout; some pulses may drop from the pulse train and cause errors in the system. The laser dynamics that lead to supermode competition and pulse dropout cannot be studied using a single pulse analysis, since a single pulse cannot be dropped. Therefore, single-pulse models cannot accurately predict the limits on the desirable operating regime. Several methods for eliminating dropouts have been demonstrated experimentally [1], [10], [11]; however, stable operation could only be obtained over a limited range of pulse durations and the limits have been poorly understood. In principle, one can study the dynamics of supermode competition by studying a long string of pulses, but in practice it is not feasible even computationally to study strings that are greater than about 24 pulses in length. We have verified that computational results converge very slowly so that even 24 pulses were not enough to obtain an accurate solution, nor do the results agree well with experiments.

In this paper we introduce an innovative yet simple technique that allows us to efficiently study pulse dropouts and other dynamic effects in harmonically modelocked fiber lasers. In our simulations, we model the propagation of a limited number of pulses and a "superpulse" that represents the pulses that are not simulated individually; the technique enables us to accurately model the impact of the amplifier gain on the pulse train. We have found that the amplifier gain affects significantly the dynamics of the laser as well as its performance, defined in terms of the pulse dropout ratio and the signal-to-noise ratio. We apply our new approach to model dynamic effects such as supermode competition, pulse dropout, and multiple pulse generation in harmonically modelocked fiber lasers. Our simulation enables us to accurately find the limits on the desirable operating regime since we can model pulses with different energies and shapes that simultaneously propagate inside the laser cavity, as occurs near the boundaries of the stable operating regime. We believe that our approach will also be useful in modeling a wide variety of lasers and storage rings containing many pulses.

We have included in our model the principal physical phenomena that affect laser performance. We have modeled the propagation of pulses in a cavity that contains fibers with a nonuniform dispersion map, an erbium-doped fiber amplifier with spontaneous emission noise and saturation, and a modelocker. We have developed a new model for calculating the saturation of homogeneously broadened amplifiers [12]. We have shown by solving the Maxwell-Bloch equations that, when a broadband pulse propagates inside a homogeneously broadened amplifier, the saturation is determined by the overlap between the amplifier gain profile and the pulse spectrum rather than by the energy of the pulse, as is assumed in the conventional models [6], [7], [13], [14]. Since an erbium-doped fiber amplifier is essentially homogeneously broadened [15] our model was used to accurately model the saturation in our laser.

We have applied our theoretical approach for studying a laser with the sigma configuration [1] and have obtained excellent quantitative agreement between the theoretical and experimental pulse spectra, autocorrelation traces, and dependence of the pulse duration on the laser power. We have also been able to obtain excellent agreement between the theoretical and the experimental autocorrelation traces of the noise surrounding the pulse and to calculate the standard deviation of the pulse energy, frequency, and jitter. We have found that an actively modelocked fiber laser can pass through four different operating regimes as the intracavity power increases; three of these regimes are observed experimentally in the sigma laser. Good agreement was obtained between theory and experiment for the power boundaries of the first three operating regimes. In the first regime the intracavity power is small and the pulse amplitude fluctuates in time. In the second regime, the Kerr effect becomes important as the intracavity power increases, leading to pulse shortening and pulse dropout. When the power increases further the laser enters a third regime, a stable mode of operation with very few pulse dropouts. Finally, still higher powers force the laser into the forth regime, in which pulse pairs are generated in some time slots. The fourth regime imposes an upper power limit upon the regime of stable pulse generation. In the sigma laser the last regime is not observed, however, because dispersion management of the laser cavity significantly increases the power at which the fourth regime occurs, making it nearly unobservable experimentally.

We have also studied the stability of the energy of a single pulse that propagates in the cavity using Lyapunov's method, assuming that all the pulses have the same amplitude and shape. Prior work for studying the stability of modelocked fiber lasers [7] and storage ring [6] were performed using soliton perturbation theory. Lyapunov's method is simpler than soliton perturbation theory, yet it gives more general and more accurate results. In particular, we could study the stability of pulses with arbitrary shapes rather than hyperbolic-secant, as is assumed in soliton perturbation theory. We found that pulses are stable only when their duration is shorter than a threshold value that depends on the mode-locker the amplifier, and the pulse shape [22]. Therefore, when the laser is insufficiently pumped it generates a limited number of pulses with dropouts in some time slots. When the repetition rate increases, the minimum average power that is needed for stable operation sharply increases. We have validated these results by comparison to complete simulations and have found that the prediction of the parametric dependence of the threshold is quite good, although there are quantitative differences.

\section{LASER AND MODEL DESCRIPTION}

\section{A. Experimental Configuration}

The laser studied experimentally was an actively mode-locked sigma laser [1]. The laser configuration, shown in Fig. 1, contains a single-polarization branch [16], allowing the incorporation of nonpolarization-maintaining fibers into a laser which acts essentially as a polarization-maintaining (PM) unidirectional ring laser. Linearly polarized light is injected from a PM fiber loop through a polarizing beamsplitter into a non-PM branch; the polarization state of the light evolves as it propagates through the branch. A $45^{\circ}$ Faraday rotator and mirror at the end of the branch returns light in a polarization state orthogonal to that of the incident light, and (for linear propagation) the polarization state of the returning light is orthogonal to that of the incident light at every point 


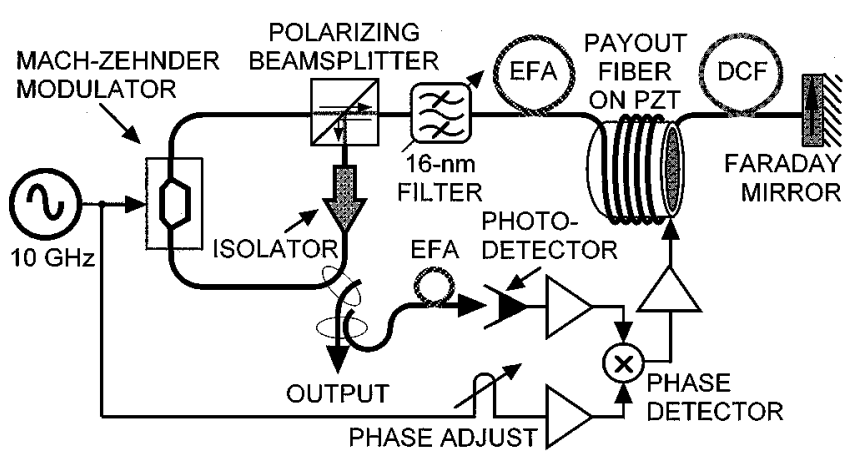

Fig. 1. Schematic description of the laser configuration that was used in the experiments. EFA is an erbium-doped fiber amplifier and DCF is dispersion compensating fiber.

in the branch. Light exiting the branch into the polarizing beamsplitter is linearly polarized and rotated by $90^{\circ}$ from the incident light. The orthogonally polarized counterpropagating light compensates birefringence variations, a major source of environmental instability in non-PM lasers. The non-PM branch contains a tunable filter with a 3-dB bandwidth of 16 $\mathrm{nm}, 12 \mathrm{~m}$ of $\mathrm{Yb}$ : Er gain fiber, $60 \mathrm{~m}$ of payout fiber (reduced dispersion fiber that can be rotated around a drum with a small diameter without adding a significant loss), and $13 \mathrm{~m}$ of dispersion-compensating fiber (DCF). The loop also contains an isolator, an output coupler, and a Mach-Zehnder intensity modulator driven by a microwave synthesizer.

A feedback system compares the phase of the laser pulse train with the synthesizer output to generate an error signal. This signal is amplified, integrated, and fed to a piezoelectric cylinder, around which is wound the payout fiber, to regulate the cavity length. The effective length of the laser is $192 \mathrm{~m}$, corresponding to a fundamental repetition rate of $1 \mathrm{MHz}$. When driven at $10 \mathrm{GHz}$, the laser operates at a harmonic order number of $10^{4}$.

\section{B. Laser Model}

The laser configuration in the theoretical model, shown in Fig. 2, is similar to that used in the sigma laser [1]. The dispersion map contained $60 \mathrm{~m}$ of payout fiber with $D_{1}=$ $13.38 \mathrm{ps} / \mathrm{nm}-\mathrm{km}, 26 \mathrm{~m}$ of dispersion compensating fiber with $D_{2}=-92 \mathrm{ps} / \mathrm{nm}-\mathrm{km}, 60 \mathrm{~m}$ of payout fiber with $D_{3}=13.38$ $\mathrm{ps} / \mathrm{nm}-\mathrm{km}$, and $46 \mathrm{~m}$ of fiber with $D_{4}=17.7 \mathrm{ps} / \mathrm{nm}-\mathrm{km}$. The theoretically estimated average dispersion at the operating wavelength of $1560.5 \mathrm{~nm}$ was $D_{\text {avg }}=0.14 \pm 0.01 \mathrm{ps} / \mathrm{nm}-\mathrm{km}$. This estimate was obtained by requiring optimum agreement between theory and experiments; due to uncertainties in estimating the cavity losses, the accuracy of the theoretical estimation of the average dispersion was limited to about 0.01 $\mathrm{ps} / \mathrm{nm}-\mathrm{km}$. The theoretical average cavity dispersion should be compared with the experimental estimate $D_{\text {avg }}=0.15$ $\mathrm{ps} / \mathrm{nm}-\mathrm{km}$, obtained by assuming that the pulse duration increases rapidly when $D_{\text {avg }}$ crosses through zero as the operating wavelength is tuned from the anomalous to the normal regime. The nonlinear coefficient of the dispersion-compensating fiber was taken to be $\gamma_{\mathrm{nl}}=4.0 \mathrm{~W}^{-1} \mathrm{~km}^{-1}$, while the nonlinear coefficient of the other fibers was $\gamma_{\mathrm{nl}}=2.1 \mathrm{~W}^{-1} \mathrm{~km}^{-1}$.

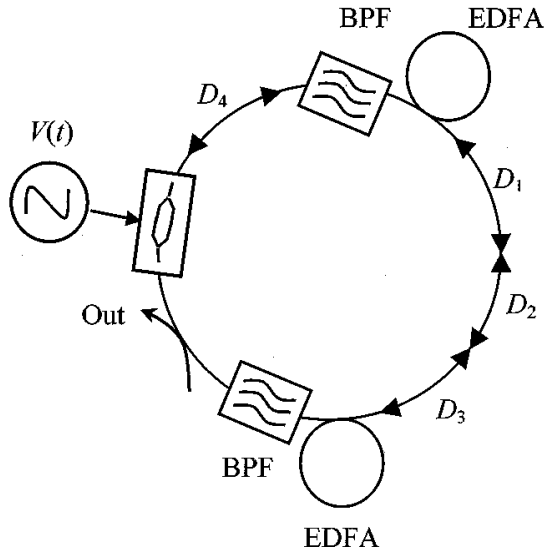

Fig. 2. Schematic description of the laser configuration that was analyzed theoretically. EDFA is an erbium-doped fiber amplifier, BPF is an optical bandpass filter, and $D_{i}$ are fibers with a dispersion coefficient $D_{i}$ and a nonlinear effect.

We have also studied theoretically and experimentally a similar laser configuration with a higher average dispersion of 0.22 $\mathrm{ps} / \mathrm{nm}-\mathrm{km}$. The length of the dispersion-compensating fiber in this configuration was $25.8 \mathrm{~m}$ while the other fiber lengths were unchanged. In order to analyze the effect of dispersion management on the sigma laser we have also modeled a laser with a uniform dispersion map that generates pulses with similar energies and durations as the sigma laser.

We calculated the pulse propagation in the fiber by solving the Manakov equation [17] using the split-step Fourier method [18]. We found that when the pulse duration was longer than about 1.1 ps, the nonlinear polarization rotation could be neglected, and the pulse propagation could be analyzed using the scalar nonlinear Schrödinger equation. In our simulation we used a ring rather than the sigma configuration in order to simplify the amplifier modeling. The optical amplifier in a laser with the sigma configuration amplifies two waves that propagate in opposite directions. Using the split-step Fourier method we could only study the propagation in a single direction and therefore we had to separately analyze the propagation of the two counterpropagating waves inside the amplifier. Since both waves affect the gain saturation of the amplifier and since the waves are also coupled due to the cavity mirror, one should strictly speaking use an iterative procedure in order to model the amplifier saturation. If a unidirectional ring cavity is modeled instead, the wave propagates in a single direction and the amplifier model therefore becomes significantly simpler. The use of a unidirectional ring rather than the full geometry of the sigma cavity does not significantly affect the amplifier model, as the excellent quantitative agreement between theory and experiment indicates. This result is not surprising because the amplifier operates in a highly saturated regime where the amplifier gain is determined by the cavity loss.

We assume an internal loss of $5 \mathrm{~dB}$ at the mode-locker, $1 \mathrm{~dB}$ at each of the two ends of the amplifier, $1 \mathrm{~dB}$ due to the polarizing beam splitter, $2 \mathrm{~dB}$ due to an output coupler that was put at the end of the amplifier, and $1 \mathrm{~dB}$ due to the other optical components. These losses in the laser cavity cause a strong variation of the power and, consequently, the strength of the nonlinear effect 
inside the cavity. The mode-locker transmissivity $T_{m}$ was taken as $T_{m}(t)=0.5\left[1-\cos \left(\omega_{m} t\right)\right]$ where $f_{m}=\omega_{m} / 2 \pi=10 \mathrm{GHz}$ and the optical bandpass filer was Lorentzian with a full width at half-maximum of $16 \mathrm{~nm}$, located between the amplifier and the output coupler.

Approximately $N=10000$ pulses simultaneously propagate inside the laser cavity. Since it is only possible to model the propagation of $n=5$ to 24 pulses, and since we found that computational results converge very slowly so that even 24 pulses were not enough to obtain an accurate solution, we added a superpulse that represents the $N-n$ pulses that are not simulated individually. The cavity energy $E_{t}$ was given by: $E_{t}=U(N-n) E_{s}+\sum_{i=1}^{n} E_{i}$ where $E_{s}$ is the energy of the superpulse and $\sum_{i=1}^{n} E_{i}$ is the sum of the energies of the ordinary pulses in the simulation. The shape of the superpulse was updated using the shape of the ordinary pulses. The update of the superpulse shape was done by replacing the shape of the superpulse with the average shape of one of the pulses that remains in the cavity. We cannot use an average over several pulses because their phases are different, but we verified that the result does not depend on which pulse we choose as long as the pulse is not dropping out. The averaging was performed over a time period that starts at the previous update of the superpulse. Typically, we updated the superpulse every 2000 roundtrips, and we confirmed that the results are not sensitive to this choice. In order to model pulse dropout we added a variable $U$ representing the fraction of the pulses that remain in the cavity. The value of $U$ was self-consistently determined in our simulation. It was updated each time that we changed the shape of the superpulse by requiring that the energy of the superpulse, which represents the total energy of all the pulses that are not kept in the simulation, remains constant. The use of a superpulse guarantees that the saturation energy of the amplifier is not strongly affected by one of the ordinary pulses, so that some of the ordinary pulses drop out while others equilibrate with the proper pulse shape. We have carefully validated this approach by gradually increasing the number of simulated pulses, and we found that the results typically converge beyond $n=5$ pulses.

Since the superpulse represents $U(N-n)$ pulses and the response time of the erbium-doped amplifier is slow, the noise from different pulses is averaged and does not strongly affect the amplifier saturation. Therefore, the density of the amplifier noise amplitude for the superpulse was taken to be smaller by a factor of $[U(N-n)]^{1 / 2}$ relative to that of the ordinary pulses.

\section{Amplifier Model}

The erbium-doped fiber amplifier in our laser was modeled as an ideal three-level system with a full width at half-maximum of $20 \mathrm{~nm}$ and a small signal gain of $25 \mathrm{~dB}$. The frequency dependence of the absorption and the gain cross sections [19] were not included in the model and instead a Lorentzian lineshape was used. This choice was justified by two facts. First, the pulse bandwidth is much narrower than that of the amplifier and therefore only the quadratic term in the frequency expansion of the gain is important. Second, the operating wavelength is around $1560 \mathrm{~nm}$ and the effect of the gain peak around 1530 $\mathrm{nm}$ is therefore small. The results of our model were insensitive to this choice for the gain profile. The length of the ampli- fier was $12 \mathrm{~m}$ and the effective filter bandwidth of the amplifier was estimated to be $17 \mathrm{~nm}$. The response time of the amplifier was estimated as $100 \mu$ s and independent of the intracavity power. We modeled the propagation in the amplifier by solving the Ginzburg-Landau equation [13] using a split-step Fourier method.

The amplifier saturation was modeled using a new model that takes into account the dependence of the saturation of the signal spectrum [12]. We have shown by solving the Maxwell-Bloch equations for a homogeneously broadened amplifier that the saturation of the amplifier is determined by the overlap between the pulse spectra and the amplifier gain profile and not by the pulse energy, as is often assumed [6], [7], [13], [14]. The new model significantly changes the effective filtering in the amplifier and it therefore affects the pulse duration predicted by our model. For ultrashort pulses that are generated by passively modelocked fiber lasers the new model also predicts a significantly higher power than that calculated using pulse energy saturation of the amplifier.

Assuming that a train of pulses $\left\{E_{i}(\omega, z)\right\}_{i=-\infty}^{i=+\infty}$ propagate along the amplifier, where $z$ is the location along the amplifier and $E_{i}(\omega, z)$ is the spectrum of the $i$ th pulse, we can calculate the gain coefficient for the $i$ th pulse at a location $z$, using [12]

$$
\begin{gathered}
g_{i+1}(z)=g_{0}+\left[g_{i}(z) \exp \left(\frac{\epsilon_{0}}{\hbar} \int_{-\infty}^{+\infty} \chi_{\mathrm{I}}(\omega)\left|E_{i}(\omega, z)\right|^{2} d \omega\right)\right. \\
\left.-g_{0}\right] \exp \left(-T / T_{1}\right)
\end{gathered}
$$

where

$\begin{array}{ll}g_{i}(z) & \text { gain coefficient for the } i \text { th pulse; } \\ g_{0} & \text { small signal gain; } \\ T_{1} & \text { population relaxation time; } \\ T & \text { time interval between pulses; } \\ \omega & \text { frequency; } \\ \epsilon_{0} & \text { electric permeability; } \\ z & \text { location along the amplifier; } \\ \chi_{\mathrm{I}}(\omega) \quad \text { imaginary part of amplifier susceptibility that is } \\ \end{array}$ The spontaneous emission noise was simulated as white noise
enerated and amplified along the whole amplifier length, in order to take into account the dependence of the noise components on the frequency. In an erbium-doped fiber amplifier atoms are pumped into a high energy manifold and then drop immediately to an intermediate quasistable energy manifold. Amplification is attained due to a transition between the intermediate and the ground state manifolds. The gain coefficient $g$ is proportional to the population difference $N_{2}-N_{1}$ [18]-[20], where $N_{2}$ and $N_{1}$ are the population density of the atoms in the intermediate and the ground states, respectively; the spontaneous emission power density is proportional to $N_{2}$. Assuming the atomic population in the uppermost energy level is negligible, the small signal gain $g_{0}$ is proportional to the total density of atoms, $N_{0}=N_{1}+N_{2}$. Using the relation $N_{2}=\left[N_{0}+\right.$ $\left.\left(N_{2}-N_{1}\right)\right] / 2$ we can calculate the spectral power density of the spontaneous emission $S_{n}(z)$

$$
S_{n}(z)=h \nu G(\nu)\left[g_{0}(z)+g(z)\right] / 2
$$



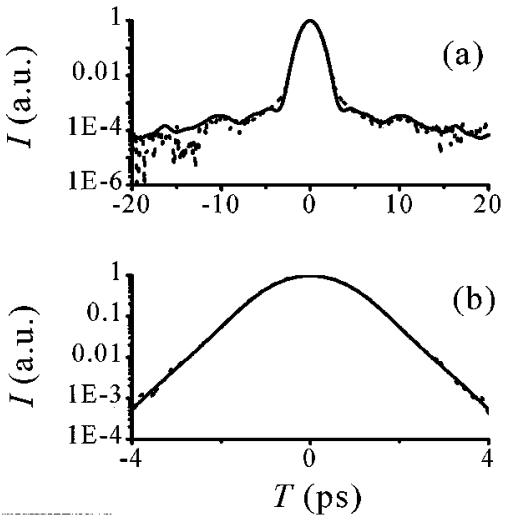

Fig. 3. Comparison between the theoretical and the experimental autocorrelation traces for a sigma laser with an average dispersion of (a) $D_{\text {avg }}=0.14 \mathrm{ps} / \mathrm{nm}-\mathrm{km}$ and (b) $D_{\text {avg }}=0.22 \mathrm{ps} / \mathrm{nm}-\mathrm{km}$.
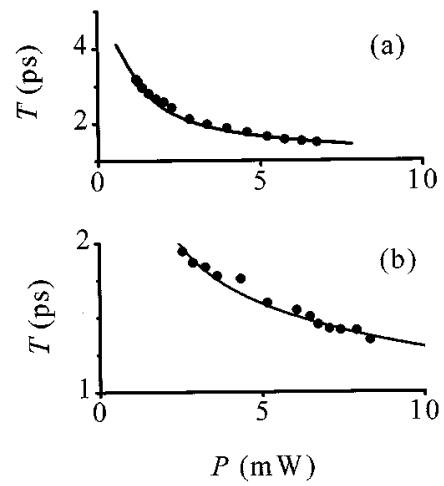

Fig. 4. Comparison between the theoretical and the experimental dependence of the full width at half-maximum of the pulse duration versus the power at the output of the coupler for a sigma laser with an average dispersion of (a) $D_{\text {avg }}=0.14 \mathrm{ps} / \mathrm{nm}-\mathrm{km}$ and (b) $D_{\text {avg }}=0.22 \mathrm{ps} / \mathrm{nm}-\mathrm{km}$

where $\nu$ is the signal frequency and $G(\nu)$ is the gain profile. Note that instead of using the spontaneous emission factor $n_{s p}$ we use a more accurate model that gives the variation of the spontaneous emission density along the amplifier. The model also predicts strong noise when the amplifier is strongly saturated and the gain is small, since an erbium-doped fiber amplifier is a three-level system and therefore more than half of the atomic population must be pumped in order to obtain net gain.

\section{Single Pulse CharaCteristics}

In order to verify that we have included in our model the main physical phenomena that affect our laser, we have compared the theoretical and the experimental autocorrelation traces, pulse spectra, and the dependence of the pulse duration on the power. The results, shown in Figs. 3-5 were obtained for a laser with a) an average dispersion of $D_{\text {avg }}=0.14 \mathrm{ps} / \mathrm{nm}-\mathrm{km}$ and b) an average dispersion of $D_{\text {avg }}=0.22 \mathrm{ps} / \mathrm{nm}-\mathrm{km}$. The figures demonstrate that our model does obtain an excellent quantitative agreement between theory and experiment. The comparison of the theoretical and the experimental autocorrelation traces also yields a good quantitative agreement of the noise levels around the pulse. We believe, therefore, that the noise model used is valid.

Our simulation results indicate that when the average dispersion is $0.14 \mathrm{ps} / \mathrm{nm}-\mathrm{km}$ and the pulse duration is about 1.4
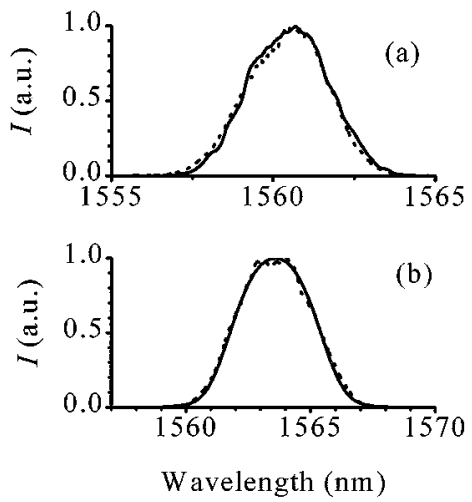

Fig. 5. Comparison between the theoretical and the experimental pulse spectra for a sigma laser with an average dispersion of (a) $D_{\text {avg }}=0.14 \mathrm{ps} / \mathrm{nm}-\mathrm{km}$ and (b) $D_{\text {avg }}=0.22 \mathrm{ps} / \mathrm{nm}-\mathrm{km}$.

ps, the standard deviation of the jitter is $32 \mathrm{fs}$, the normalized standard deviation of the pulse energy is $1.0 \%$, the normalized standard deviation of the pulse amplitude is $1.3 \%$ and the standard deviation of the average pulse wavelength is $0.05 \mathrm{~nm}$. The central pulse time, $t_{p}$ was calculated using the relation [23], $t_{p}=\int_{-\infty}^{\infty} t|u|^{2} d t / W$ and the central pulse frequency $\Omega$, was calculated using the relation, $\Omega=\int_{-\infty}^{\infty}\left(u_{t} u^{*}-u_{t}^{*} u\right) / 2 i W$, where $W$ is the pulse energy, $u$ is the pulse amplitude, and $u_{t}$ is the time derivative of $u$. The measured jitter in the sigma laser was less than $10 \mathrm{fs}$ over a frequency range of $100 \mathrm{~Hz}-1 \mathrm{MHz}$ [5]. The jitter was measured in the experiments by converting the phase noise of the laser into an amplitude noise via mixing the detected pulse train with a low-noise synthesizer [24]. The jitter is proportional to the total power of the phase noise [24]. The lowest frequency components of the phase noise of the laser could not be measured due to $1 / f$ phase noise originating in the synthesizer; therefore, the theoretical and the experimental results cannot be easily compared.

The sigma laser uses a strong dispersion-management scheme. We have studied theoretically the effect of dispersion management on the laser noise by comparing the noise in the sigma laser to the noise in a laser with a uniform dispersion map and a dispersion coefficient $D=1.2 \mathrm{ps} / \mathrm{nm}-\mathrm{km}$. The loss map in the uniform dispersion laser was the same as in the sigma laser. The value of $D$ was chosen in order to obtain the same output power in the uniform dispersion laser and in the sigma laser at a pulse duration of $1.4 \mathrm{ps}$. The jitter in the laser with uniform dispersion map was about $120 \mathrm{fs}$, the energy noise was about $0.65 \%$, and the noise level around the pulse (the "continuum") was about $10 \mathrm{~dB}$ higher than in the sigma laser. The decrease in the jitter of the sigma laser is due mainly to the decrease in the average cavity dispersion, as is the case in optical transmission systems [23]. The pulse energy is stabilized in soliton lasers since changes in the pulse energy affect the pulse bandwidth and hence affect the cavity loss for that pulse due to the effective amplifier bandwidth [6]. In the laser with the uniform dispersion map this feedback was stronger and therefore the energy noise was slightly weaker than in the sigma laser; however, due to the increase in the continuum level in the uniform-dispersion laser, the improvement in the amplitude noise was relatively small. A more detailed 
discussion of the effect of dispersion management on the laser operation is given in [25].

\section{THE Four OPERATING REGIMES}

Our theoretical approach enables us to study the propagation of several pulses with different energies and shapes inside the laser as appropriate to high-repetition rate fiber lasers that are used in optical communication systems. Therefore, we can accurately study dynamic effects that limit stable operation of fiber lasers, such as pulse dropout or supermode competition. A single-pulse analysis cannot accurately find the limits on the stable operating regime, since close to the limits of this regime pulses with different energies and temporal profiles simultaneously propagate in the cavity and since the dependence of the number of pulses on the average cavity power exhibits a bistable behavior.

We found that a laser with a uniform dispersion map passes through four different operating regimes as the laser power changes; three of these regimes are observed experimentally in the sigma laser. The last operating regime was not observed in the sigma laser. This laser contains strong dispersion management which significantly increases the power at which the last regime occurs, making it nearly unobservable experimentally and theoretically. Therefore, the first three operating regimes were studied in the sigma laser, while the last regime was modeled for a laser with a uniform dispersion map.

\section{A. First Regime-Linear Evolution}

When the energy inside the cavity is very low, nonlinear effects can be neglected. Fig. 6(a) shows the pulse train in this case. In this first regime the results of the simulation indicate that the pulses are noisy, exhibiting amplitude and pulse shape fluctuations. This noisy behavior, which leads to supermode competition in the frequency domain, is due to the lack of stabilization from nonlinear effects, so that the pulse amplitude can change without affecting its bandwidth. The temporal pulse shape is approximately Gaussian with an average full width at half-maximum of $4.5 \mathrm{ps}$ consistent with the $4.4 \mathrm{ps}$ Kuizenga-Siegman limit [26]. The transition between this regime and the next is smooth and is caused by the amplified spontaneous emission noise that limits the coherence time of the laser. Without this noise, the nonlinearity always becomes important on a sufficiently long time scale and leads to pulse dropout even when the intensity is extremely low. We obtained this result by running long simulations without including the spontaneous emission noise and by performing a stability analysis using our reduced model, described below. The noise limits the coherence length of the laser and the time over which the nonlinear effect can accumulate. The transition to the next operating regime will therefore occur only when the pulse power is sufficiently large that the nonlinear length is shorter than the coherence length of the laser. In this case the nonlinear effect will lead to pulse dropout and transition to the second operating regime as described below. We note that when the power level is small enough so that even a single pulse is not affected by the nonlinearity, then no pulse dropout occurs even without including the spontaneous emission noise [6]. This
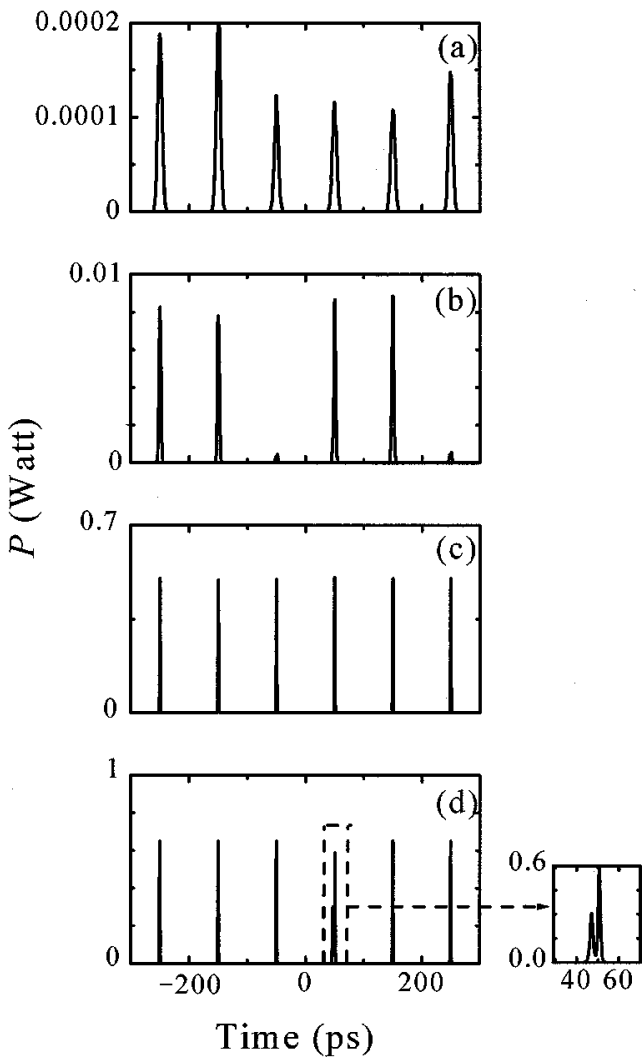

Fig. 6. Pulse train for an output power of (a) $P=14 \mu \mathrm{W}$, (b) $2 \mathrm{~mW}$, (c) 32 $\mathrm{mW}$, obtained for a laser with a sigma configuration and an average dispersion $D_{\text {avg }}=0.14 \mathrm{ps} / \mathrm{nm}-\mathrm{km}$. Pulse train for an output power of (d) $P=42 \mathrm{~mW}$ obtained for a laser with a uniform dispersion map with a dispersion coefficient $D=1.2 \mathrm{ps} / \mathrm{nm}-\mathrm{km}$. Six pulses are simulated and the first pulse is the superpulse. The results displayed here were obtained after 150000 iterations.

issue is important in lasers that only support a small number of pulses, but in the laser that we are studying, which contains about 10000 pulses, this power level is around $50 \mathrm{nW}$; so, this issue is not important.

Due to the significant cavity loss, $\approx 11 \mathrm{~dB}$, the optical power changes significantly with position inside the cavity. Therefore, we define our intracavity power $P$ at a specific location-the input end of the output coupler. In fact, the power in the nonpolarization maintaining branch, where most fibers are located, is around 2.6 times weaker than the power at the input end of the coupler.

Noisy pulse generation in the first operating regime is demonstrated for the sigma laser in Fig. 7(a). The figure presents sampling oscilloscope density contour plots of the output of the laser, as detected by a $\sim 20 \mathrm{GHz}$ photodiode, at two intracavity powers $P$ (measured at the input end of the output coupler). The left trace was taken at the lowest power measured; the right trace was taken at a power near the upper boundary of the first regime. The filled-in traces indicate large amplitude fluctuations in the pulse. Fig. 8 presents the measured pulse duration $\tau_{1 / 2}$, the 3 -dB bandwidth $\Delta \nu$, and duration-bandwidth product $\tau_{1 / 2} \Delta \nu$ for the sigma laser over the measured range of intracavity powers. Since the autocorrelation trace of the pulse resembles a Gaussian autocorrelation trace more than a hyperbolic-secant trace as is often assumed, we estimated the pulse duration from the measured autocorrelation trace assuming that 


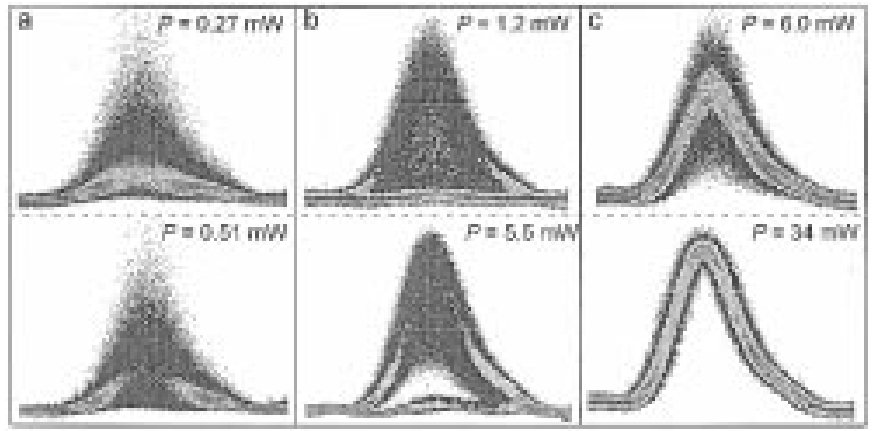

Fig. 7. Sampling oscilloscope contour density plots of the detected pulses formed by the sigma laser as a function of the intracavity average power $P$. Three of the four predicted regimes of operation are observed: (a) the linear pulse production regime, showing noisy pulse production; (b) the low-power nonlinear regime, in which the number of dropouts decreases as $P$ increases; (c) dropout-free pulse production. In the last regime the amplitude noise can be seen to decrease with increasing $P$.

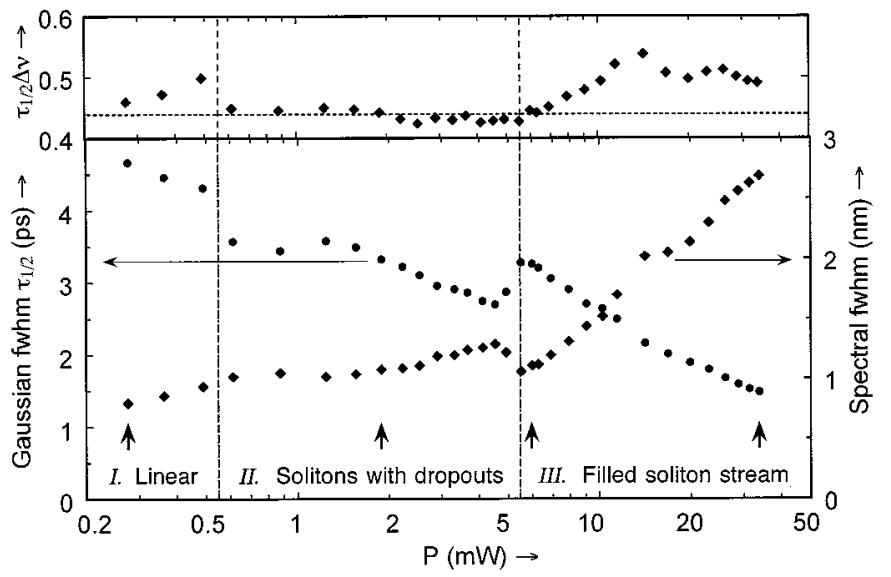

Fig. 8. The pulse duration, optical bandwidth, and the duration-bandwidth product $\tau_{1 / 2} \Delta \nu$ of the pulses produced by the sigma laser as a function of the intracavity average power $P$. The vertical dotted lines divide the graph into the three observed regimes of operation; the horizontal dotted line indicates the transform-limited duration-bandwidth product of 0.44 for Gaussian pulses.

the pulse shape has a Gaussian profile. In the first operating regime $\tau_{1 / 2}$ ranges from $4.7-4.4$ ps and $\tau_{1 / 2} \Delta \nu$ is greater than the transform limit of 0.44 for Gaussian pulses.

The theoretical intracavity power $P$ at the boundary between the first and the second operating regimes is about $0.45-0.55$ $\mathrm{mW}$. This transition occurs over a broad power regime and the power at the boundary between the regimes strongly depends on the noise in the laser. The power at the boundary between the regimes measured experimentally is about $0.6 \mathrm{~mW}$. This result as well as the smooth behavior of the transition that was observed in the experiments are in a good agreement with the theoretical results.

\section{B. Second Regime-Nonlinear Evolution with Pulse Dropout}

In the second regime, shown in Fig. 6(b), nonlinear effects become important and decrease the pulse duration from about 3.9 ps (full width at half-maximum) to about 2.9 ps at the upper boundary of this regime. The experimental results, shown in Fig. 8 indicate that the duration-bandwidth product is very close to the transform limit for Gaussian pulses, even though the pulse duration decreases from $3.6 \mathrm{ps}$ at the lower boundary of this regime to $2.7 \mathrm{ps}$ at the upper boundary.

The direct interaction between the cavity pulses is weak in actively modelocked lasers due to the mode-locker; however, the pulses all interact indirectly via the amplifier. Due to the very slow response time of the Er-doped amplifier, the pulses all affect the amplifier saturation, causing some pulses to drop out, while the remaining pulses will possess nearly identical shapes and amplitudes. Pulses drop out due to a positive feedback caused by the gain saturation and the mode-locker. When the pulse duration is long the cavity loss due to the mode-locker is higher than the loss caused by the effective amplifier bandwidth. For such pulses, an increase of the energy in one of the pulses leads to a decrease in its duration due to nonlinear effects, increasing the transmissivity of the mode locker for that pulse, and leading to a further increase in its energy. Eventually, the pulse duration will decrease and will reach its steady-state value due to the saturation of the amplifier gain and due to the increase of the loss caused by the effective amplifier optical filtering. Pulse dropout is caused by the amplifier saturation. The increase of the energy of some pulses saturates the amplifier gain until the gain becomes equal to the loss for those pulses. The pulses that maintain their original duration will have higher loss in the mode-locker and will therefore experience net loss in a roundtrip; they will eventually disappear. The decrease of the amplifier gain due to the decrease of the loss for pulses that remain in the cavity will prevent unstable growth of noise and/or new pulses in the spaces where pulses previously dropped, in contrast to the prediction for a system that only contains a single pulse or a stream of pulses that is exactly periodic [6].

Fig. 9 shows the energy, the duration, and the mode-locker transmissivity for a pulse that is dropped and for a pulse that continues to propagate in the cavity. It also shows the average amplifier gain. Six pulses were simulated; the first pulse is the superpulse. It is assumed that at $t=0$ all the other pulses have the same amplitude. The transmissivity of the mode-locker for a pulse is defined as the energy of the pulse at the output of the element divided by the energy at the input. The average transmissivity of the amplifier is defined as the constant cavity loss multiplied by the ratio between the average output energy and the average input energy of the amplifier. The results shown in Fig. 9 were calculated for a laser with a uniform dispersion map with a dispersion coefficient of $D=2 \mathrm{ps} / \mathrm{nm}-\mathrm{km}$. In this laser the average dispersion is higher than in the sigma laser and the pulse dropout is obtained at significantly higher powers. Therefore, the effect of the noise is smaller than in the sigma laser while the effect of the nonlinearity on the pulse parameters is stronger, making it easier to observe the pulse dropout dynamics.

Note the large fluctuations, on a long time scale, in the energy of the pulse that remains in the cavity, shown in Fig. 9. These slow fluctuations are caused by the slow response time of the amplifier. The change in the energy of the pulses affects the amplifier gain only after a relatively long time. Until the amplifier reaches its steady state gain, the energies of all the pulses undergo large fluctuations.

Fig. 7 shows sampling oscilloscope contour density plots for two pulses near the lower and upper intracavity power boundaries of the second regime. The traces demonstrate that dropouts 

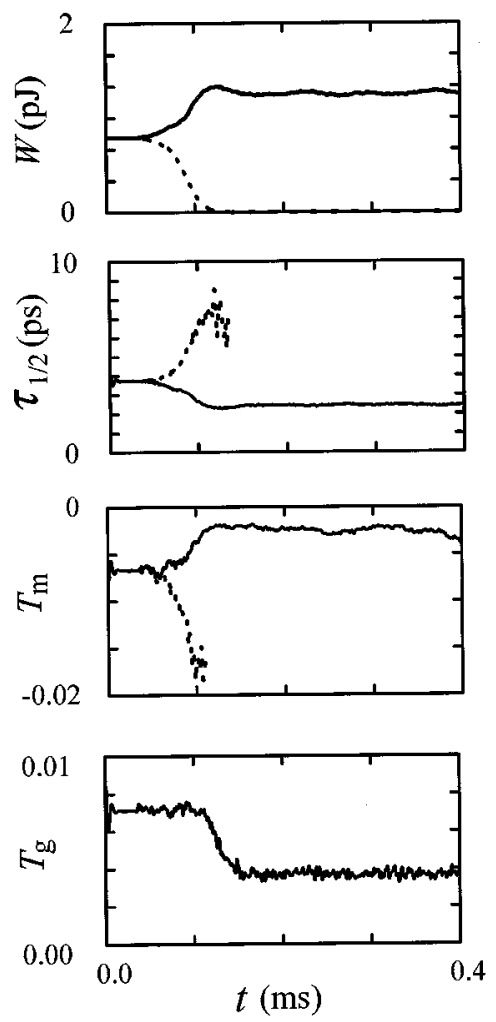

Fig. 9. Theoretical results for the pulse energy $W$, duration $\tau_{1 / 2}$, and the transmissivity of the mode-locker $T_{m}$, for a pulse which is eventually dropped (dotted curves) and for a pulse that remains (solid curves). Also shown in the figure the average amplifier gain $T_{g}$. At $t=0$ all the six pulses that were simulated have the same amplitude and shape. The results were obtained for a laser with a uniform dispersion map and a dispersion coefficient $D=2$ $\mathrm{ps} / \mathrm{nm}-\mathrm{km}$. The average intracavity power equals $12 \mathrm{~mW}$.

occur in the pulse train; as $P$ increases, the fraction of pulses that drop out decreases. However, it is expected that the number of filled slots is not a simple function of the intracavity power but also depends on the previous state of the laser, since it is possible to remove some fraction of the pulses after they have been established. The others compensate by increasing their energies and decreasing their duration. This effect causes a bistable transition to the next operating regime where there is no dropout, as described below.

\section{Third Regime-Nonlinear Evolution Without Pulse Dropout}

Beyond $P=2.5 \mathrm{~mW}$ we enter the third regime in which all the time slots can be filled, as shown in Fig. 6(c). The amplitude of the pulses is stabilized due to the effective amplifier filtering [14]. When all time slots are filled the equilibrium pulse characteristics may be determined by the conventional analysis [8], [14]; however, inclusion of the gain dynamics allows us to calculate the low-frequency noise components due to the amplifier gain, and to analyze dynamic effects, such as the ability of the laser to recover when a pulse is removed. When the average power is below $5 \mathrm{~mW}$, some pulses may be removed, as in the second regime; however, above this power level, the system will quickly restore pulses that drop. Clearly, the latter regime is the optimal regime in which to operate a laser. By contrast, fiber storage rings, which are in many ways analogous to lasers [14], [29] should not work in this regime. The approach described in this paper should allow the user to determine the fraction of pulses that may be safely removed from a storage ring with fixed intracavity power. We would also note that we obtained in our simulations strong noise at very low frequencies (on the order of tens of $\mathrm{kHz}$ ) that are due to relaxation oscillations.

The theoretical simulations indicate that the minimum power needed for stable operation without dropouts is about $2.5 \mathrm{~mW}$. A practical laser, however, should also be able to recover from random dropouts due to changes in the environmental conditions. The minimum power needed for the laser to recover from random pulse dropout is about $5 \mathrm{~mW}$. This result is in good quantitative agreement with the experimentally measured lower limit of the stable operating regime- $-5.5 \mathrm{~mW}$.

When the laser power increases above the minimum power needed to recover from dropouts the pulse duration sharply increases from about 2.9-3.4 ps, since when the missing pulses are restored, the number of pulses increases, and the pulse energy decreases. The experimental results, shown in Fig. 7(c) and 8 , indicates that the pulse duration increases abruptly from 2.7-3.3 ps through the transition between the second and the third regime. The pulse duration decreases smoothly to a minimum observed value of $1.4 \mathrm{ps}$ as $P$ increases to its maximum value of $34 \mathrm{~mW}$. The duration-bandwidth product increases above the Gaussian limit as $P$ increases in this regime. Fig. 7 also demonstrates that the amplitude noise decreases as $P$ increases.

The difference between the minimum power needed for stable operation and the power needed to recover from a dropout causes a bistable transition between the second and the third operating regimes that was observed both experimentally and theoretically. When approaching the transition to the third regime from below, some pulses are missing, and the laser must possess sufficient intracavity power to restore the missing pulses. When approaching the transition from above, dropout-free operation will be maintained as long as the pulse energies are greater than the minimum power needed for stable propagation of the pulses. The model predicts that a bistable region might exist between cavity powers $P=2.5$ and 5.0 $\mathrm{mW}$. In the bistable region the laser can theoretically operate without dropouts and therefore this regime is included in the third operating regime. In practice, the lower power of the bistable region strongly depend on the number of pulses in the cavity and on the effect of the environmental conditions on the stability of the pulses. Environmental perturbations can significantly narrow this bistable region; in the sigma laser, it extends only over a power range of approximately $5 \%$.

The lowest power that is needed for stable operation can be estimated for our laser with fair accuracy using Lyapunov's method [27], [28]. This methods predicts the stability condition of one of the pulses propagating inside the laser cavity, assuming that all the pulses have the same amplitude and shape [22]. Because our stability analyzes ignores many-body effects, its accuracy is limited; however, it is computationally rapid and allows its user to explore a wide parameter range. Stability analysis for solitons propagating inside a storage ring has been performed in previous work using soliton perturbation theory [6], based on modifications of the Ginzburg-Landau equation [8], [9]. Our approach gives more general and accurate results with a little 
additional effort. We calculate the energy change in each of the cavity elements without using the assumptions needed to derive the modified Ginzburg-Landau equation and without assuming that the dependence of the pulse energy on the pulse duration is the same as in the case of hyperbolic-secant solitons. We need only assume that the full width at half-maximum, $\tau$, is a decreasing function of the pulse energy, $W$.

The maximum value of the full width at half-maximum $\tau_{\max }$ possible for stable operation is given by [22]

$$
\tau_{\max }=\left(\frac{2 G\left\langle\omega^{2}\right\rangle}{\left\langle x^{2}\right\rangle M \omega_{m}^{2} \omega_{g}^{2}}\right)^{1 / 4}
$$

where

$\begin{array}{ll}G=g_{0} l_{g} /(1+ & \text { average gain coefficient; } \\ \left.\ln T_{0}+2 g_{0} l_{g}\right) & \\ T_{0} & \begin{array}{l}\text { energy transmissivity of the laser cavity } \\ \text { that does not depend on the pulse; }\end{array} \\ & \text { amplifier length; } \\ l_{g} & \text { small signal gain; } \\ g_{0} & \text { modulation frequency; } \\ f_{m}=\omega_{m} / 2 \pi & \text { amplifier bandwidth; } \\ \omega_{g} & \text { modulation depth; } \\ M & \int_{-\infty}^{\infty}|f(x)|^{2} x^{2} d x \int_{-\infty}^{\infty}|f(x)|^{2} d x, \\ \left\langle x^{2}\right\rangle= & \left\langle\omega^{2}\right\rangle \\ & \int_{-\infty}^{\infty}|d f(x) / d x|^{2} d x / \int_{-\infty}^{\infty}|f(x)|^{2} d x, \\ & \text { and } f(t / \tau) \text { is the pulse profile at the } \\ & \text { mode-locker and the amplifier. }\end{array}$

For a Gaussian pulse the coefficient $\left\langle\omega^{2}\right\rangle /\left\langle x^{2}\right\rangle$ equals $[4 \ln (2)]^{2}$.

The minimum average intracavity power, needed for stable operation, approximately equals

$$
P_{\min }=\frac{\operatorname{sech}^{-1}\left(2^{-1 / 2}\right) E D_{\mathrm{avg}}}{\pi \gamma_{\mathrm{nl}}} \omega_{m}^{3 / 2}\left(\frac{8 M \omega_{g}^{2}\left\langle x^{2}\right\rangle}{G\left\langle\omega^{2}\right\rangle}\right)^{1 / 4}
$$

where $D_{\text {avg }}$ is the average dispersion and $E$ is the energy enhancement factor [30], [31]. When the intracavity power is less than $P_{\text {min }}$, the laser generates a limited number of short and intense pulses with a duration shorter than $\tau_{\max }$, while other pulses are dropped from the pulse train. Note that the minimum power sharply increases when the repetition rate increases due to the increase of the pulse number (proportional to $\omega_{m}$ ), the increase in the mode-locker effect (proportional to $\omega_{m}^{1 / 2}$ ), and the increase in the energy enhancement factor, which strongly depends on $\tau_{\max }$ [30], [31].

Assuming that the pulse shape is Gaussian, the average dispersion $D_{\text {avg }}$ is $0.14 \mathrm{ps} / \mathrm{nm}-\mathrm{km}$, and the dispersion map strength factor $\gamma$ [30], [31] equals 6.2 for pulses with a 1.4 ps duration we can use (4) and the connection between the $E$ and $\gamma$ [30], [31], and obtain, $\tau_{\max }=3.1 \mathrm{ps}$ and $P_{\min }=3.0 \mathrm{~mW}$ at the input end of the coupler. This result is in a good agreement with the results obtained by our full model, $\tau_{\max }=3.5 \mathrm{ps}$ and $P_{\min }=2.5$ $\mathrm{mW}$. We note the result of (4) was adjusted in order to take into account the transmission of the coupler and the change in the power along the cavity due to losses. We assumed that the power at the input end of the output coupler is about 2.6 times higher than in the non polarization-preserving branch where most of the dispersion map is located.

\section{Fourth Regime-Multiple Pulse Generation}

In some harmonically mode-locked laser configurations, a fourth operating regime exists in which more than one pulse occupies a single time slot. In order to clearly observe the fourth operating regime we modeled a laser with a uniform dispersion map and a dispersion coefficient $D=1.2 \mathrm{ps} / \mathrm{nm}-\mathrm{km}$. When the pulse duration equals $1.4 \mathrm{ps}$ and the laser performance is optimal, the output power in the uniform dispersion laser and in the sigma laser are the same. The fourth operating regime is shown in Fig. 6(d). The figure shows that when the output power equals $42 \mathrm{~mW}$, a pair of pulses is generated in some of the time slots. The interaction between the two pulses continuously changes their energy and shape; these changes lead to noise in the other pulses. The energy in a pulse pair is about 1.7 times larger than in a single pulse. This ratio is less than two since a mutual pulse interaction prevents obtaining an optimum transmissivity for the pulse pair in the mode-locker. In our laser model the transition from a single to a complex pulse is as sharp as in the case of passively modelocked lasers [32]. The formation of pulse pair limits the minimum pulse duration in this laser to about 1 ps. In previous work, Kärtner et al. [7] found an analytical limit on the minimum pulse duration due to the generation of a continuum around the pulse. The difference between the limits stems at least in part from our inclusion of more than one pulse in the model. Therefore, a small excess of energy in several pulses may accumulate in a single pulse to form a pulse pair rather than a continuum around each pulse.

The formation of pulse pairs is due to excess loss from amplifier bandwidth filtering and from nonlinear effects. As the energy of a pulse increases so does its bandwidth. Since the transmissivity of the mode-locker does not change significantly on a time scale on the order of the pulse duration, at some point it becomes energetically favorable to generate two pulses with a narrower bandwidths in the same time slot. Nonlinear effects also promote the generation of pulse pairs since single pulses have higher intensities and therefore they are deteriorated and experience higher loss in the mode-locker. In fact, when the laser power was slightly lower than the boundary between the third and the fourth operating regimes we observed in the simulation results an ordered modulation in the pulse envelope, similar to that caused by the modulational instability effect on solitons [33].

We found both theoretically and experimentally that the fourth operating regime did not appear in the sigma laser for the highest power $P$ that was investigated in our simulations -150 $\mathrm{mW}$ - and for the maximum power that was available in the experiment $-34 \mathrm{~mW}$. However, the simulations indicate that as the power increases, the pulse shape deteriorates and the bandwidth increases similar to what occurs in optical transmission systems [31]. We have also found out that when the power is excessive there is also an increase in the intensity of the pulse tails and the noise level ("continuum") around the pulse that may limit the use of such pulses in optical communication systems. 


\section{CONCLUSIONS}

We have analyzed an harmonically modelocked fiber laser that employs the Kerr effect in order to shorten the pulse duration. In order to analyze this laser system, which contains about $10^{4}$ pulses in its cavity, we used an innovative numerical approach, based on the use of a superpulse and a new model for the amplifier. By carefully modeling the main physical phenomena that affect the laser we obtained an excellent quantitative agreement between the theoretical and the experimental results for a laser with a sigma configuration. Four different behavior regimes were theoretically observed, depending on the intracavity power, in a laser with a uniform dispersion map; three of the regimes were observed experimentally in the sigma laser. The use of a dispersion map in the sigma laser significantly broadens the stable operating regime and decreases the jitter and the noise around the generated pulses ("continuum").

\section{REFERENCES}

[1] T. F. Carruthers and I. N. Duling III, "10-GHz, 1.3-ps erbium fiber laser employing soliton pulse shortening," Opt. Lett., vol. 23, pp. 1927-1929, 1996.

[2] M. L. Dennis, T. F. Carruthers, W. I. Kaechele, R. B. Jenkins, J. U. Kang, and I. N. Duling III, "Long span repeaterless transmission using adiabatic solitons," IEEE Photon. Technol. Lett., vol. 11, pp. 478-480, 1999.

[3] M. L. Dennis, W. I. Kaechele, L. Goldberg, T. F. Carruthers, and I. N. Duling III, "Wavelength-division-multiplexed $4 \times 10 \mathrm{~Gb} / \mathrm{s}$ adiabatic soliton transmission over 235 km," IEEE Photon. Technol. Lett., vol. 11, pp. 1680-1682, 1999.

[4] G. M. Carter, R.-M. Mu, V. S. Grigoryan, C. R. Menyuk, P. Sinha, T. F. Carruthers, M. L. Dennis, and I. N. Duling III, "Transmission of dispersion-managed solitons at $20 \mathrm{Gbit} / \mathrm{s}$ over $20000 \mathrm{~km}$," Electron. Lett., vol. 35, pp. 233-234, 1999.

[5] T. R. Clark, T. F. Carruthers, P. J. Matthews, and I. N. Duling III, "Phase noise measurements of ultrafast $10 \mathrm{GHz}$ harmonically modelocked fiber laser," Electron. Lett., vol. 35, pp. 720-721, 1999.

[6] H. A. Haus and A. Mecozzi, "Long-term storage of a bit stream of solitons," Opt. Lett., vol. 17, pp. 1500-1502, 1992.

[7] F. X. Kärtner, D. Kopf, and U. Keller, "Solitary-pulse stabilization and shortening in actively mode-locked lasers," J. Opt. Soc. Amer. B, vol. 12, pp. 486-496, 1995.

[8] H. A. Haus, "A theory of forced mode locking," IEEE J. Quantum Electron., vol. 11, pp. 323-330, 1975.

[9] D. E. Martinez, R. L. Fork, and J. P. Gordon, "Theory of passively mode-locked lasers for the case of a nonlinear complex-propagation coefficient," J. Opt. Soc. Amer. B, vol. 2, pp. 753-760, 1985.

[10] N. Nakazawa, K. Tamura, and E. Yoshida, "Supermode noise suppression in a harmonically modelocked fiber laser by selfphase modulation and spectral filtering," Electron. Lett., vol. 32, pp. 461-463, 1996.

[11] G. T. Harvey and L. F. Mollenauer, "Harmonically mode-locked fiber ring laser with an internal Fabry-Perot stabilizer for soliton transmission," Opt. Lett., vol. 18, pp. 108-110, 1993.

[12] M. Horowitz, C. R. Menyuk, and S. Keren, "Modeling the saturation induced by broadband pulses amplified in an erbium-doped fiber amplifier," J. Lightwave Technol., vol. 11, pp. 1235-1237, 1999.

[13] G. P. Agrawal, "Amplification of ultrashort solitons in erbium-doped fiber amplifiers," IEEE Photon. Technol. Lett., vol. 2, pp. 875-877, 1990.

[14] H. A. Haus and A. Mecozzi, "Noise of mode-locked lasers," IEEE J. Quantum Electron., vol. 29, pp. 983-996, 1993.

[15] E. Desurvire, J. L. Zyskind, and J. R. Simpson, "Spectral gain holeburning at $1.53 \mu \mathrm{m}$ in erbium-doped fiber amplifiers," IEEE Photon. Technol. Lett., vol. 2, pp. 246-248, 1990.
[16] I. N. Duling and R. D. Esman, "Single-polarization fiber amplifier," Electron. Lett., vol. 28, pp. 1126-1128, 1992.

[17] D Marcuse, P. K. A Wai, and C. R. Menyuk, "Application of the Manakov-PMD equation to studies of signal propagation in optical fibers with randomly varying birefringence," J. Lightwave Technol., vol. 15, pp. 1735-1746, 1997.

[18] G. P. Agrawal, Nonlinear Fiber Optics. San Diego: Academic, 1995, ch. 1.

[19] E. Desurvire, "Study of the complex atomic susceptibility of erbiumdoped fiber amplifiers," J. Lightwave Technol., vol. 8, pp. 1517-1527, 1990.

[20] A. Yariv, Optical Electronics. New York: Holt, Rinehart, and Winston, 1985, pp. 123-135.

[21] G. P. Agrawal, Optical Communication Systems. New York: WileyInterscience, 1997, ch. 1.

[22] M. Horowitz and C. R. Menyuk, "Analysis of pulse dropout in harmonically modelocked fiber lasers using Lyapunov's method," Opt. Lett., vol. 25 , pp. 40-42, 2000.

[23] V. S. Grigoryan, C. R. Menyuk, and R.-M. Mu, "Calculation of timing jitter and amplitude jitter in dispersion-managed optical fiber communications using linearization," J. Lightwave Technol., vol. 17, pp. 1347-1356, 1999.

[24] D. von der Linde, "Characterization of the noise in continuously operating mode-locked lasers," Appl. Phys. B, vol. 39, pp. 201-217, 1986.

[25] T. F. Carruthers, I. N. Duling III, M. Horowitz, and C. R. Menyuk, "Dispersion management in a harmonically mode-locked fiber soliton laser," Opt. Lett., vol. 25, pp. 153-155, 2000.

[26] D. J. Kuizenga and A. Siegman, "FM and AM mode locking of the homogeneous laser-part I: Theory," IEEE J. Quantum Electron., vol. 6, pp. 694-706, 1970.

[27] J.-J. E. Slotine and W. Li, Applied Nonlinear Control. Englewood Cliffs, NJ: Prentice-Hall, 1991, pp. 53-57.

[28] N. Minorsky, Nonlinear Oscillation. New York: D. Van Nostrand, 1987.

[29] M. F. Nakazawa, E. Yamada, H. Kubota, and K. Suzuki, "10 Gbit/s soliton data transmission over one million kilometers," Electron. Lett., vol. 27, pp. 1270-1272, 1991.

[30] N. J. Smith, N. J. Doran, W. Forysiak, and F. M. Knox, "Soliton transmission using periodic dispersion compensation," J. Lightwave Technol., vol. 10, pp. 1808-1822, 1997.

[31] V. S. Grigoryan, T. Yu, E. A. Golovchenko, C. R. Menyuk, and A. N. Pilipetskii, "Dispersion-managed soliton dynamics," Opt. Lett., vol. 22, pp. 1609-1611, 1997.

[32] A. B. Grudinin, D. J. Richardson, and D. N. Payne, "Energy quantization in figure eight fiber laser," Electron. Lett., vol. 28, pp. 67-68, 1992.

[33] J. P. Gordon, "Dispersive perturbations of solitons of the nonlinear Schrödinger equation,” J. Opt. Soc. Amer. B, vol. 9, pp. 91-97, 1992.

Moshe Horowitz, photograph and biography not available at the time of publication.

Curtis R. Menyuk (F'98), photograph and biography not available at the time of publication.

Thomas F. Carruthers, photograph and biography not available at the time of publication.

Irl N. Duling, III (M'97), photograph and biography not available at the time of publication. 